ARTICLE

\title{
Structural basis of the P4B ATPase lipid flippase activity
}

Lin Bai ${ }^{1,4 凶}$, Bhawik K. Jain (1D ${ }^{2,4}$, Qinglong You (D) ${ }^{3,4}, \mathrm{H}$. Diessel Duan (1) ${ }^{3}$, Mehmet Takar (D) ${ }^{2}$, Todd R. Graham (1D) ${ }^{2 凶} \&$ Huilin Li (iD ${ }^{3 凶}$

P4 ATPases are lipid flippases that are phylogenetically grouped into P4A, P4B and P4C clades. The P4A ATPases are heterodimers composed of a catalytic $\alpha$-subunit and accessory $\beta$-subunit, and the structures of several heterodimeric flippases have been reported. The $S$. cerevisiae Neo1 and its orthologs represent the P4B ATPases, which function as monomeric flippases without a $\beta$-subunit. It has been unclear whether monomeric flippases retain the architecture and transport mechanism of the dimeric flippases. Here we report the structure of a P4B ATPase, Neo1, in its E1-ATP, E2P-transition, and E2P states. The structure reveals a conserved architecture as well as highly similar functional intermediate states relative to dimeric flippases. Consistently, structure-guided mutagenesis of residues in the proposed substrate translocation path disrupted Neo1's ability to establish membrane asymmetry. These observations indicate that evolutionarily distant P4 ATPases use a structurally conserved mechanism for substrate transport.

\footnotetext{
${ }^{1}$ Department of Biochemistry and Biophysics, School of Basic Medical Sciences, Peking University, Beijing, China. ${ }^{2}$ Department of Biological Sciences, Vanderbilt University, Nashville, TN, USA. ${ }^{3}$ Department of Structural Biology, Van Andel Institute, Grand Rapids, MI, USA. ${ }^{4}$ These authors contributed

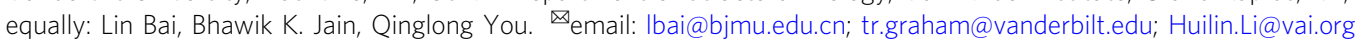


T he phospholipid bilayer of biological membranes provides a fundamental barrier that encloses the cell and most internal organelles, while integral membrane proteins allow for the selective passage of ions and polar molecules. The lipid organization of many membranes is asymmetric. For example, the plasma membrane of mammalian cells contains more phosphatidylethanolamine (PE) and phosphatidylserine (PS) in its cytosolic leaflet, and more phosphatidylcholine (PC) and sphingolipids in the extracellular leaflet ${ }^{1-3}$. Phospholipid asymmetry is crucial for many cellular processes, including vesicular transport, signal transduction, cell motility, cell polarity, cell division, and the transport of ions and other molecules across the bilayer ${ }^{1,4-7}$. Phospholipid asymmetry is regulated by three types of transporters: scramblases, floppases, and flippases ${ }^{8,9}$. Scramblases catalyze a nonselective, bidirectional, trans-bilayer movement of the phospholipid down their concentration gradients. Floppases belong to the ATP Binding Cassette (ABC) transporter family and translocate phospholipids to the extracellular leaflet of membranes. Most flippases belong to the type IV P-type ATPase (P4 ATPase) family and selectively transport phospholipids to the cytosolic leaflet of membranes.

P-type ATPases form ATP-dependent phosphorylated intermediates during their transport of substrates across biological membranes ${ }^{10}$. Based on sequence homology, P-type ATPases are divided into five subclasses (P1-P5) ${ }^{11-14}$, and each subclass typically transports different substrates. The P1, P2, and P3 ATPases mainly transport cations (e.g., the $\mathrm{Ca}^{2+}$-ATPase, $\mathrm{Na}^{+} / \mathrm{K}^{+}$-ATPase, and the proton pump) or heavy metals (e.g., the copper transporter). In stark contrast, the substrates of P4 ATPases are bulky phospholipid molecules, and these transporters are further divided into $\mathrm{P} 4 \mathrm{~A}, \mathrm{P} 4 \mathrm{~B}$, and $\mathrm{P} 4 \mathrm{C}$ clades $^{15}$. The $\mathrm{P} 5$ ATPases transport polyamines or transmembrane helices ${ }^{16-19}$. Most P-type ATPases have a conserved architecture consisting of a transmembrane domain (TMD), an actuator domain (A-domain), a nucleotide-binding domain ( $\mathrm{N}$-domain), and a phosphorylation domain (P-domain) ${ }^{12}$. According to the structural studies of the most well-understood P2 ATPases, P-type ATPases transport their substrate through a cyclic transition of E1-E1P-E2P-E2 states, which was called the "Post-Albers" model ${ }^{20-24}$. This cycle starts with the binding of ATP to the E1 state followed by autophosphorylation to form E1P. The P4-ATPases do not require transport substrate interaction to generate the E1P or E2P intermediates; however, lipid substrate binding induces dephosphorylation of the pump and is associated with translocation of lipid across the bilayer ${ }^{25}$.

Saccharomyces cerevisiae has five P4 ATPases-Drs2, Neo1, Dnf1, Dnf2, and Dnf3, each having a different subcellular location and substrate specificity-whereas humans have $14^{26-28}$. Notably, most P4 ATPases transport phospholipids as a heterodimeric complex, with the major catalytic subunit referred to as the $\alpha$ subunit and the partner protein as the $\beta$-subunit. The $\beta$-subunit is essential for the stability and trafficking of the flippase, as well as for substrate binding ${ }^{29-32}$. For P4A ATPases, the $\alpha$-subunit makes extensive contacts with the $\beta$-subunit on the extracellular side of the membrane, within the membrane, and on the cytosolic side. These $\alpha \beta$ interactions are thought to be crucial for the distinct conformation dynamics of P4 ATPases that control lipid translocation. However, the yeast Neol and its related human proteins (ATP9A and ATP9B) are monomeric, and the structural basis for how they function without the accessory $\beta$-subunit has been unclear.

Neo1 was first identified as a neomycin-resistance-1 gene (NEO1) that prevented aminoglycoside toxicity when overexpressed ${ }^{33}$. Neo1 is essential for cell growth and is found in the Golgi and endosomes, where it regulates membrane trafficking ${ }^{34-37}$. Unlike other P4 ATPases, the flippase activity of Neol has not been demonstrated with purified protein yet. However, temperature-sensitive alleles of NEO1 were found to cause a loss of PE and PS asymmetry in the plasma membrane but with a greater influence on PE exposure ${ }^{38}$. Depletion of TAT-5, the Caenorhabditis elegans ortholog of Neo1, leads to exposure of only $\mathrm{PE}$ in the extracellular leaflet, suggesting the P4B-ATPases play a more substantial role in establishing PE asymmetry ${ }^{39}$. Although it lacks a $\beta$-subunit, Neol is regulated by Dop1 (Dopey ortholog), Mon2 (which is a relative of large Arf guanine nucleotide exchange factors), the small GTPase Arll, and Any1 $34,40-43$. In fact, Neo1, Mon2, Arl1, and Dop1 assemble into a membrane remodeling complex ${ }^{40,41}$. These regulatory proteins are crucial for yeast growth and likely interact with Neol only transiently with low binding affinity, but little is known about their functions. The disruption of $A N Y 1$, which encodes a PQ-loop membrane protein, can rescue the growth deficiency of a $\Delta$ neol or $\Delta d r s 2$ strain $^{38,43,44}$.

Recently, several P4A ATPase structures have been reported, including the $S$. cerevisiae Drs2-Cdc50 $0^{45,46}$, Dnf1-Lem $3^{32}$, Dnf2-Lem $3^{32}$, the C. thermophilum Dnf1-Cdc5047, the human ATP8A1-CDC50A ${ }^{25}$, and ATP11C-CDC50A ${ }^{48}$. These structural studies revealed a conserved architecture among the P4 ATPases, including the 10-helix TMD, the cytosolic A domain, $\mathrm{N}$ domain, and P domains, and a conserved ATP-dependent lipid transport cycle. Several of the P4A ATPase structures contain substrate lipid-bound in a lumenal or exoplasmic "entry site" formed from residues that had previously been implicated in substrate recognition through mutagenesis studies $32,48-50$. A second substrate lipid-binding site on the cytosolic side of the membrane, an exit site, has been identified only in the Dnf1 and Dnf2 structures ${ }^{32,47}$. Surprisingly, this large exit gate extends $10 \AA$ out of the bilayer, causing the membrane to dimple into the cytosol, and it uses an Arg residue from the N-terminal cytosolic domain of the $\beta$-subunit to help coordinate the lipid headgroup ${ }^{32}$. However, it has been unclear whether the monomeric lipid flippase Neo1 functions by a similar mechanism or whether the cytosolically exposed exit gate is a conserved aspect of the substrate translocation path for P4 ATPases that lack a $\beta$-subunit.

An important question is how the substrate specificity is achieved for the P4B ATPases. Inactivation of Neo1 perturbs the PS and PE asymmetry of the plasma membrane, but whether or not Neo1 interacts directly with these lipids as transport substrates are unclear. By comparison, Drs2-Cdc50 primarily transports PS, while Dnf1-Lem3/Dnf2-Lem3 primarily transports glucosylceramide (GlcCer), PC, and PE, and a small amount of $\mathrm{PS}^{51-53}$. The lipid substrate specificity of P4A ATPases appears to be defined by the physiochemical properties of substrate binding pockets as demonstrated structurally and functionally $25,32,45-47,50,52,54$ However, it is not clear if the mechanism of substrate recognition and translocation is conserved for all P4-ATPases, including the evolutionarily distant P4B-APases. To address these questions, we purified the yeast Neol; examined its substrate-stimulated, in vitro ATP hydrolysis activity; determined cryo-EM structures of Neol in three intermediate states, and performed extensive structure-guided mutagenesis and functional assays. Our work identifies unique residues responsible for substrate specificity and provides insights into the conservation of the cytosolic binding site despite the lack of a $\beta$-subunit.

\section{Results and discussion}

Neo1 ATPase activity is stimulated by PE and PS. We overexpressed Neo1 in S. cerevisiae using a multicopy plasmid with a strong GAP promoter and an N-terminal triple FLAG tag. The detergent dodecyl maltoside (DDM) was used to solubilize the membrane and we purified Neol with an anti-FLAG affinity column followed by size-exclusion chromatography, during 
a

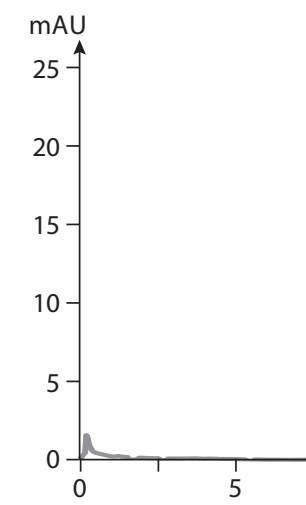

C

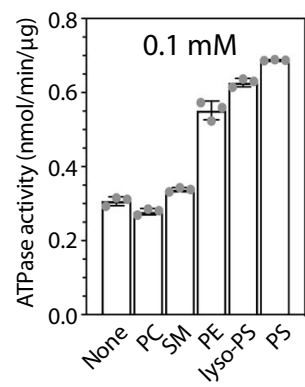

b

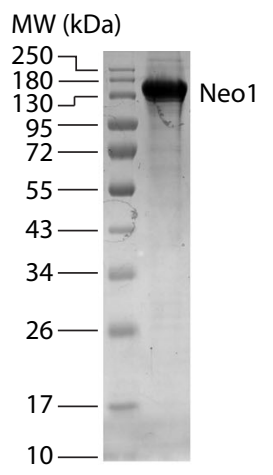

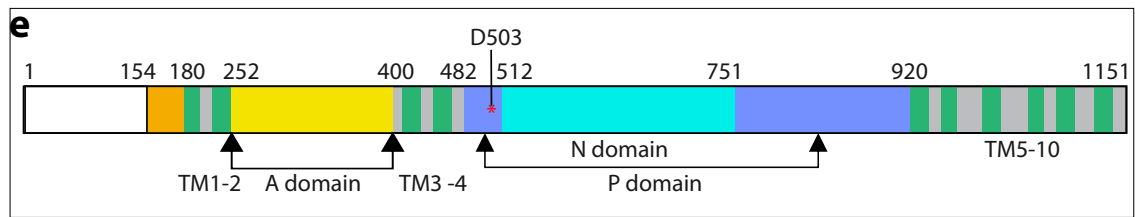

d

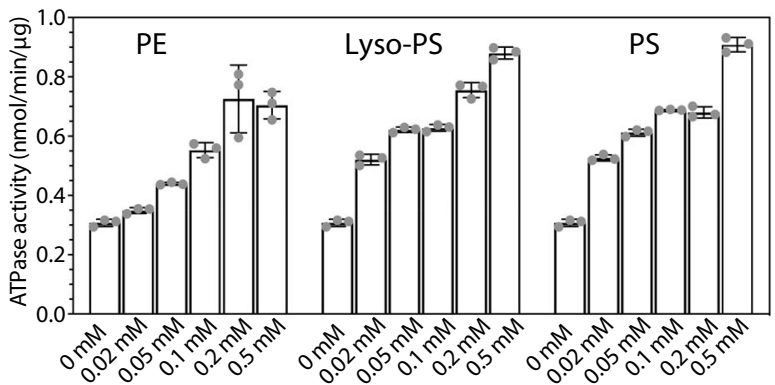

D503

Peak

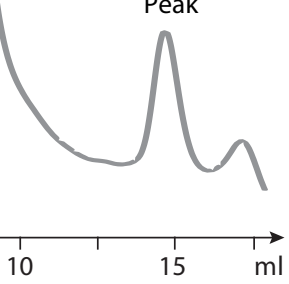


was comparable, lyso-PS had the lowest $K_{\mathrm{m}}$ that was about half of $\mathrm{PE}$, suggesting a preference for lyso-PS. In contrast, $\mathrm{PC}$ and sphingomyelin did not stimulate the ATPase activity above the low basal level detected in the absence of phospholipid. Prior studies of the influence of Neol on membrane asymmetry suggested PE was the preferred Neol substrate ${ }^{35}$, but both PS and PE effectively stimulated ATPase activity. These data showed that the purified Neol was active and that the PS, PE, and lyso-PS are likely native substrates of the flippase.

Cryo-EM structure of Neol in the E2P state. We performed single-particle cryo-EM on purified samples either directly or after incubation with different inhibitors to capture structures in different states. We obtained cryo-EM 3D maps of Neol in the E1-ATP state stabilized by AMPPCP at $5.6-\AA$ resolution, in the E2P-transition state of dephosphorylation stabilized by $\mathrm{AlF}_{4}{ }^{-}$at the $3.1-\AA$ resolution, and in the $\mathrm{E} 2 \mathrm{P}$ state as stabilized by $\mathrm{BeF}_{3}-$ at $3.2-\AA$ resolution (Supplementary Table 1, Supplementary Figs. 1-4). The bound $\mathrm{AlF}_{4}^{-}$and $\mathrm{BeF}_{3}-$ mimic the planar pentavalent transition state of phosphoryl transfer and the covalent phosphorylated E2P ground state, respectively ${ }^{14}$. We found that 3D maps of Neo1 in the E2P-transition and E2P states were superimposable (Supplementary Fig. 5). This is consistent with the previous observation that the Dnf2-Lem3 structures in the two states are nearly indistinguishable ${ }^{32}$. We first built atomic models into the 3.2- $\AA$ resolution map in the E2P state and into the 3.1- $\AA$ resolution map in the E2P-transition state. The $3 \mathrm{D}$ maps had excellent main-chain connectivity and side-chain densities except for four disordered loops: the N-terminal tail (residues 1-153), the $\mathrm{N}$-domain loop (542-559), the P-domain loop (804-821), and the C-terminal tail (1142-1151) (Fig. 1e). We next used the E2P structure to help build an atomic model for the lower-resolution 3D map of Neol in the E1-ATP state. All these models fit well with the $3 \mathrm{D}$ maps and were refined to good statistics (Supplementary Table 1).

As shown in the E2P state structure, Neo1 contains all conserved P-type ATPase domains: a 10-TM helix (TMH1-10) TMD; the cytosolic A domain inserted between TMH2 and TMH3; and the cytosolic $\mathrm{P}$ and $\mathrm{N}$ domains that are both inserted between TMH4 and TMH5 (Fig. 1e-g). The Neol structure aligns well in both TMD and soluble domains with the a-subunits of heterodimeric P4 ATPases Dnf1-Lem3, ATP8A1-CDC50, ATP11C-CDC50, and Drs2-Cdc50 (Supplementary Fig. 6). Note that TMH1, TMH4, and TMH6 of Neol are kinked just as they are in other P4 ATPases whose structures are known $25,32,45-48$. This conserved structural feature is essential for the substrate transporting activity. The Neo1 TMH4 kink is enabled by the highly conserved Pro-456.

The $\mathrm{N}$ - and $\mathrm{C}$-terminal peptides play regulatory roles and share low sequence homology among the P4 ATPases. For example, the C-terminal peptides of the yeast Drs2 and the corresponding human homolog ATP8A1 are autoinhibitory $25,45,46,55,56$. The $\mathrm{N}$-terminal peptide of Neol interacts with the cargo-selective sorting nexin Snx3 to mediate Neo1 trafficking ${ }^{36}$. The N-terminal peptide preceding the TMH1 is 183 residues long, of which the first 153, containing the Snx3 binding site, were disordered in our structure. The 30-residue ordered region of the $\mathrm{N}$-terminal peptide (Glu-154 through Val-183) formed a short $\beta$-strand and a short $\alpha$ helix that binds to the catalytic A domain which is essential for ATP turnover. This interaction enables signal transmission from the A domain to the substrate transporting path in the TMD (Fig. 1g). Unlike other P4 ATPases, the Neol C-terminal peptide following the TMH10 (Tyr-1142 to Pro-1151) is short and disordered. Therefore, it is unlikely that the Neol C-terminal tail is an autoinhibitory domain comparable to Drs2 or ATP8A1.
Neol structure in the E1-ATP state and the conserved ATPdependent transport mechanism. In the 5.6- $\AA$ resolution $3 \mathrm{D}$ map of Neo1 in the E1-ATP state determined in the presence of $1 \mathrm{mM}$ AMPPCP (a nonhydrolyzable ATP analog), the AMPPCP density is clear and the molecule stabilizes the interface between the $\mathrm{N}$ and $\mathrm{P}$ domains (Fig. 2a, Supplementary Fig. 4). Comparison of the Neol structures in the E1-ATP and the E2P states shows that the whole TMD and the $\mathrm{P}$ domain are superimposable, while the $\mathrm{N}$ and $\mathrm{A}$ domains have undergone dramatic conformational changes (Fig. 2b). In the E1-ATP structure, the N domain is packed tightly on $\mathrm{P}$ domain through the AMPPCP ligand. The A domain is also ordered, although it has no interaction with the $\mathrm{P}$ domain and interacts only with a short helixturn-helix motif (Lys704 to Leu736) in the $\mathrm{N}$ domain. The long TMH2 bends upwards at Lys-236 away from the P domain. Transitioning from the E1-ATP state to the E2P state, ATP is cleaved, ADP is released from the $\mathrm{N}$ domain, and the phosphate is transferred onto Asp-503 of the $\mathrm{P}$ domain. The phosphorylated aspartate is mimicked by the $\mathrm{BeF}_{3}{ }^{-}$moiety in our Neol structure. To enter into the E2P state, both the $\mathrm{N}$ and A domains swing away from the membrane and pack tightly together. Accompanying this transition, the TMH2 becomes straightened, pointing downwards to allow the A domain movement. These large conformational changes are likely propagated to the substratebinding sites and alter the substrate-binding affinity, leading to the transport of a lipid molecule across the membrane. Conversely, the transition from $\mathrm{E} 2 \mathrm{P} \rightarrow \mathrm{E} 2 \rightarrow \mathrm{E} 1-\mathrm{ATP}$ should close the exit site and release substrate into the cytosolic leaflet.

We next compared the Neol E1-ATP structure with the published structure of Dnf1-Lem3 in the same state and found that the TMD and the cytosolic domains are positioned similarly (Fig. 2c). Furthermore, the conformation and the substrate transport path of Neo1 in the E2P state highly resemble those of the Drs2, Dnf1, ATP8A1, and ATP11C flippases in the same E2P state (Supplementary Fig. 6). The lack of TMH3-10 movement through the P4 ATPase transport cycle was proposed to be conferred by the tightly associated $\beta$-subunit ${ }^{25}$, but Neo1 retains this property in the absence of a stabilizing $\beta$-subunit. This observation strongly suggests that all P4- ATPases-whether functioning as a single subunit in the case of Neol or with a partner $\beta$-subunit in the majority of cases-employ a conserved substrate transport mechanism. A recent functional study of the Neo1 substrate entry site supports such conserved transport mechanism ${ }^{57}$. Therefore, the $\beta$ subunit may not be primarily functioning to constrain the conformational changes in TMD, as previously proposed ${ }^{25}$.

The substrate transport path and the substrate specificity of Neo1. Structures of six P4 ATPases have been reported so far; four are in complex with substrate lipids, including $S$. cerevisiae Dnf1-Lem3, Chaetomium thermophilum Dnf1-Cdc50, human ATP8A1-CDC50, and human ATP11C-CDC5025,32,45-48 (Supplementary Fig. 6). These structures reveal a conserved flippase architecture, similar conformational changes as the enzymes go through the transport cycle, and a very similar substrate translocation path, despite having different substrate specificities. A structure-based homology search revealed that Neol in the E2P state aligned well with all known P4 ATPase structures in that state, but the best match was with Dnf1 (Fig. 3a). Yet the superposition of Neo1 and Dnf1 revealed a conspicuous difference between these flippases: the ECL2, 4, and 5 of Dnf1, which are primarily responsible for binding the $\beta$-subunit Lem3, are much longer than those in Neo1 (Fig. 3a, b). These extracellular loops are similar in length between Neol and cation transporters. This observation led us to hypothesize that P4A ATPases evolved 

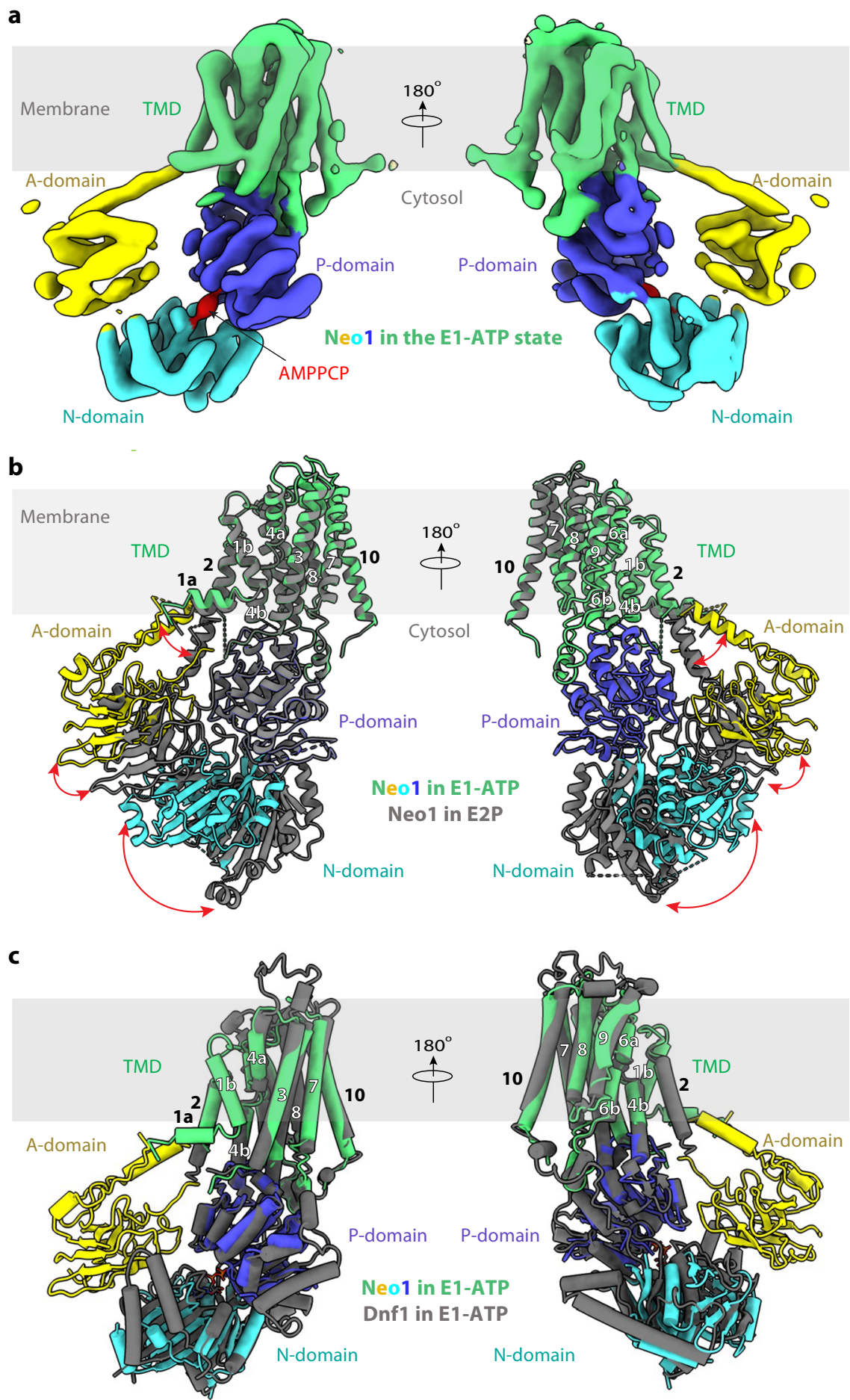

Fig. 2 Structure of the S. cerevisiae Neo1 in the E1-ATP state. a Cryo-EM 3D map of Neo1 in the E1-ATP, in the back (left) and front (right) views. The major domains are labeled in different colors. The AMPPCP density is red. $\mathbf{b}$ Structural comparison between Neo1 in the E1-ATP state (colors) and in the E2P state (gray) by aligning their respective TMDs. c Comparison of Neo1 (colors) and Dnf1 (gray) in the E1-ATP state by aligning their respective TMDs. The A domain of Dnf1 in the E1-ATP state is disordered and not modeled.

into two-subunit ATPases by the lengthening of ECL2, 4, and 5, thereby acquiring the ability to bind a $\beta$-subunit. It's also possible that the two subunits bind first, and then the a-subunit evolved these longer loops to form the stabilized complex.

Previous reported Dnf1 structure contained phospholipid molecules in both the substrate entry and exit sites, but we did not capture any endogenous lipid molecules in the Neo1 substrate sites. By aligning the Neol and Dnf1 structures, we narrowed down the substrate translocation path of Neol to a groove in the membrane domain between TMH1-4 and TMH6 (Fig. 3a, c). This putative substrate path can be divided into three sections: the top (lumenal) substrate entry site composed of several polar residues (Gln-209, Ser-221, Tyr-222, Ser-452, and Thr-453); the middle transport path between lipid-binding sites that contains the hydrophobic gate (Phe-202, Leu-226, Val-229, Val-457, and Val-461) and flanking polar residues (Gln-193, Thr-233, Arg-460, 


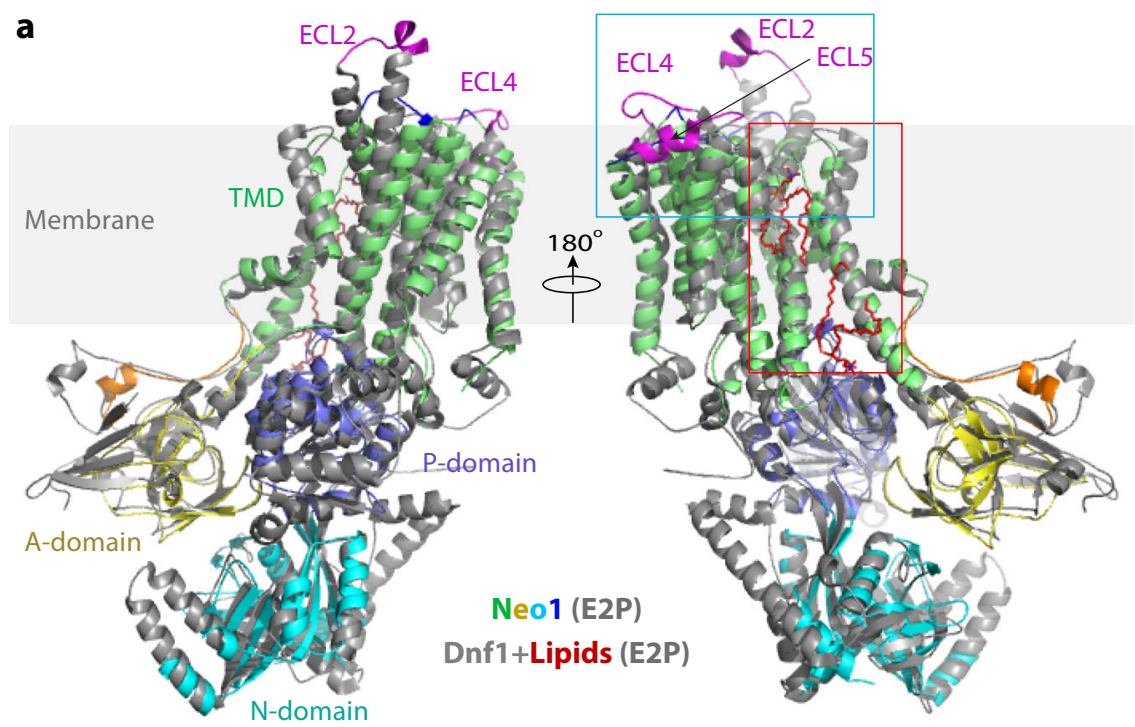

b

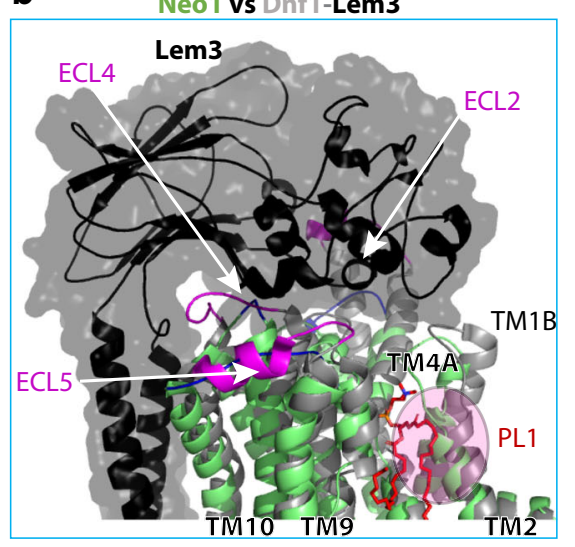

C

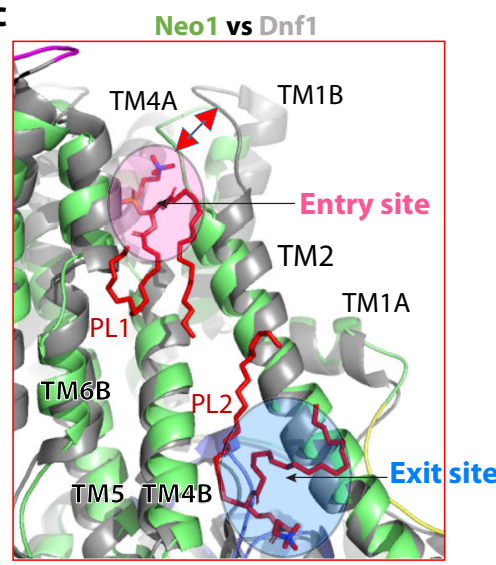

Fig. 3 Structural comparison between Neo1 and Dnf1 in the E2P state. a Lipids in the Dnf1 structure are shown as red sticks. The PL binding sites are demarcated by the red rectangle and enlarged in (c). The cyan square marks the $\beta$-subunit interacting top region of the Dnf1. All flippases have five extracellular loops (ECLs) connecting TM1 and 2, TM3 and 4, and TM5 and 6, TM7 and 8, and TM9 and 10. ECL2, 4, and 5 of Dnf1 (magenta) are much longer than those of $\mathrm{Neo1}$ (blue). b The extended ECL2, 4, and 5 of Dnf1 insert into and tightly bind the $\beta$-subunit Lem3; the corresponding loops of Neo1 are short and Neo1 lacks a $\beta$-subunit. $\mathbf{c}$ Enlarged view of the substrate transport path of Neo1 and Dnf1. The pink and blue ovals mark the lipid entry and exit sites. The double-headed red arrow marks the 7- $\AA$ outward movement of TM1B and associated ECL1 relative to Neo1, due to the presence of lipid at the entry site in Dnf1.

and Asp-464); and the bottom (cytosolic) substrate exit site lined by many polar residues (Lys-236, Asp-240, Gln-243, Arg-247, Ser-468, Glu-475, and Ser-488) (Fig. 4a). To identify key residues responsible for Neol substrate-transporting activity and specificity, we next carried out extensive structure-guided mutagenesis and functional assays (Fig. 4b, Supplementary Figs. 7 and 8). Most of these residues were mutated to either alanine or to residues present at the same position in other P4 ATPases.

Neo1 is essential for cell viability, and so we used a plasmid shuffle complementation assay to test whether Neol variants could support the viability of a neo1 $\Delta$ strain (Fig. 4b, Supplementary Fig. 7). The parental neo1 $\Delta$ strain harboring wild-type (WT) NEO1 on a URA3-marked plasmid was transformed with HIS3-marked plasmids harboring either WT Neo1, an empty vector, or the Neol mutants. The strains were replica-plated on SD medium to select for both plasmids, or on SD plus 5-FOA to counter-select against the URA3 plasmids. Because neo $1 \Delta$ cells are inviable, the empty vector control strain failed to grow on 5-FOA, while the WT copy of Neol fully supported growth. We observed that all the Neol mutants supported viability comparably to WT
Neo1 except for Neo1 P456G, which failed to support growth (Fig. 4b). Pro-456 is near the center of M4, and nearly all P-type ATPases have a proline in this position that is crucial for unwinding the M4 helix. We found that Neo1 P456G totally abolished the Neol activity in vivo. Surprisingly, Neo1 P456A fully supported viability, suggesting that maintenance of hydrophobicity with alanine in this region is essential (Fig. 4b).

Previous studies indicated that there is partial functional redundancy between Neo1 and Drs2. Therefore, we also tested the Neol mutants for their ability to support the growth of a $d r s 2 \Delta$ neo $1 \Delta$ double mutant over a range of temperatures, which provides a more sensitive assay for loss of Neol function (Fig. 4c, Supplementary Fig. 8). drs $2 \Delta$ single mutants grow well at $30^{\circ} \mathrm{C}$, but are cold-sensitive and grow slowly at $25^{\circ} \mathrm{C}$ and fail to grow at $20^{\circ} \mathrm{C}$. We found that the exit-site mutations Neo1 S488W and Neo1 R247L were lethal when combined with drs $2 \Delta$ and failed to grow at $30^{\circ} \mathrm{C}$. Many of the other Neol entry-site and transportpathway mutations substantially reduced growth in this background relative to WT Neol. These results indicate that the Neol variants represent an allelic series, ranging from complete loss 
a

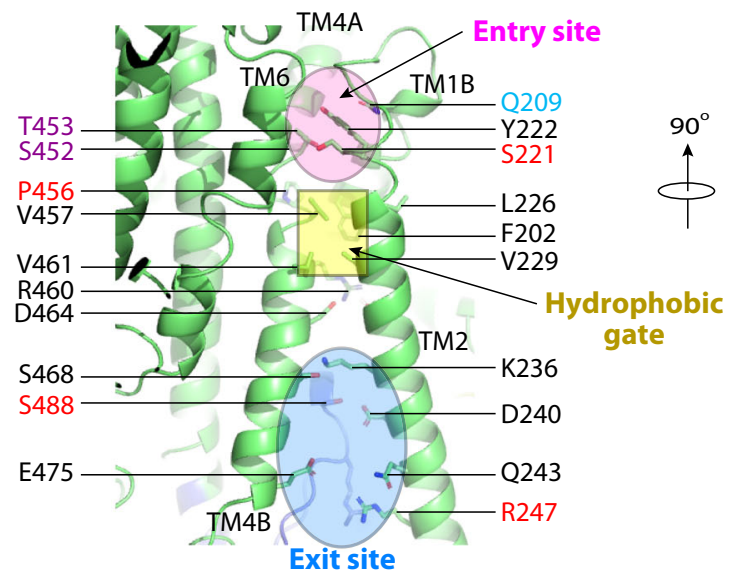

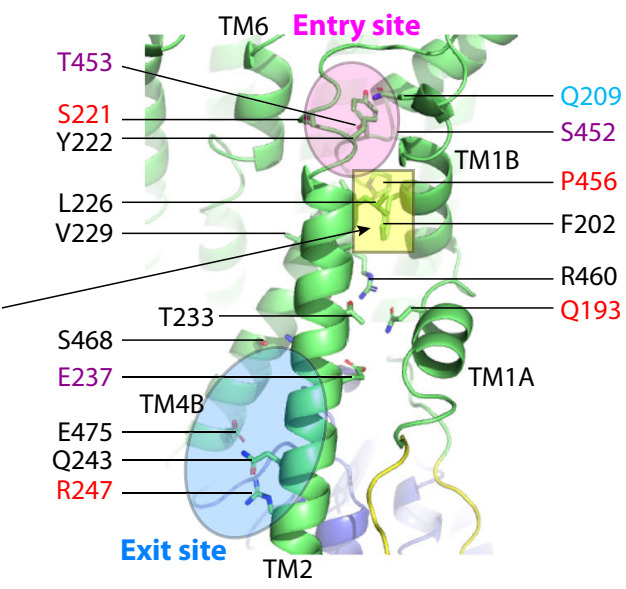

$\mathrm{P} 456 \mathrm{G}<\mathrm{S} 488 \mathrm{~W}=\mathrm{R} 247 \mathrm{~L}<\mathrm{S} 221 \mathrm{~L}<\mathrm{Q} 193 \mathrm{~A}<\mathrm{S} 452 \mathrm{Q}<\mathrm{E} 237 \mathrm{D}<\mathrm{R} 247 \mathrm{~A}<\mathrm{P} 456 \mathrm{~A}=\mathrm{T} 453 \mathrm{~S}<\mathrm{S} 488 \mathrm{~A}=\mathrm{Q} 209 \mathrm{G}$ b

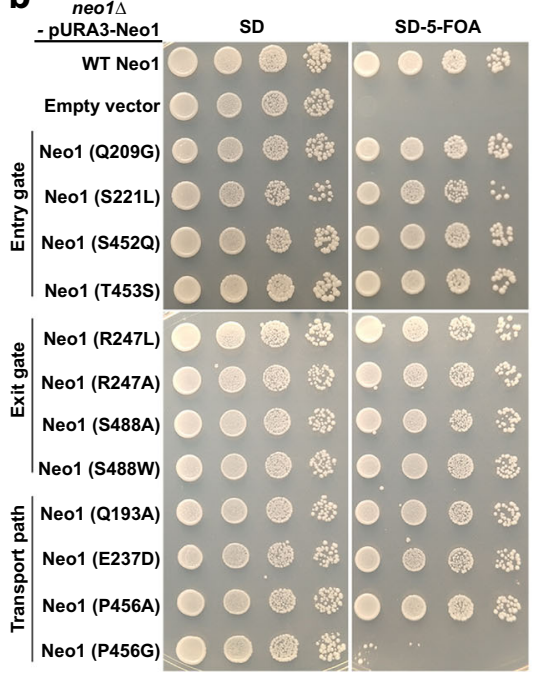

C

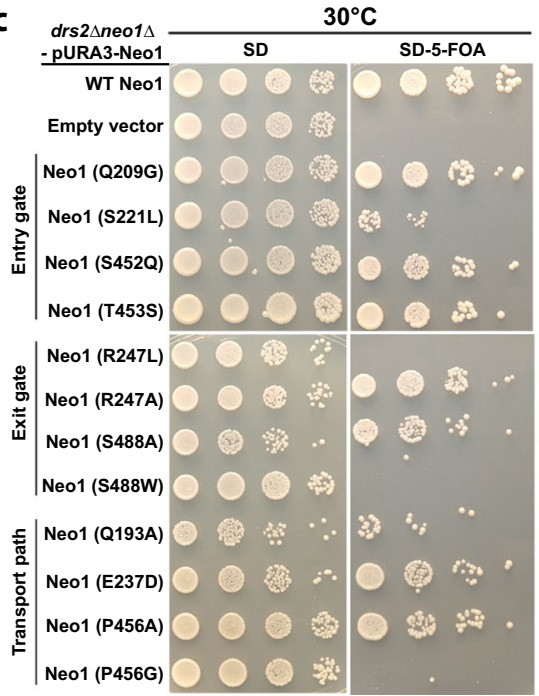

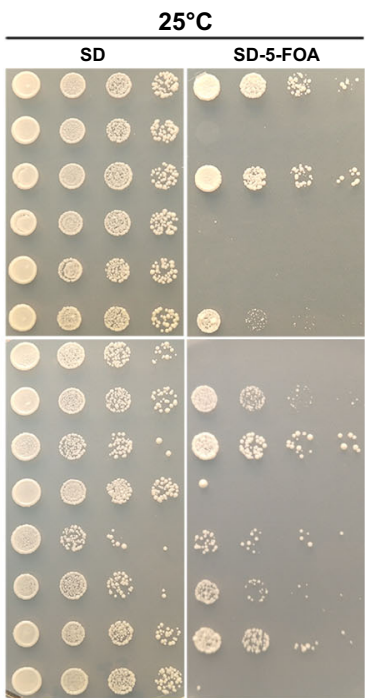

Fig. 4 Mutagenesis of the substrate path. a Putative substrate transport path of Neo1. Cartoon view of the substrate-transporting channel in the Neo1 structure with the residues lining the path shown as sticks. The individual Neo1 mutational effects based on growth phenotypes of neo1 (b) and neo1 $1 \Delta$ $\operatorname{drs} 2 \Delta$ (c) strains are summarized at the bottom of (a) with a color scheme of red (severe), purple (mild), and cyan (nearly normal). Strains on the SD medium expressed both WT and mutant variants of Neo1, but SD + 5-FOA medium eliminated WT Neo1 expressed from the URA3-marked plasmid. Colony size on the 5-FOA medium indicates how well the Neo1 variants support growth.

of function (P456G) to apparent WT activity, with respect to cell viability $\quad(\mathrm{P} 456 \mathrm{G}<\mathrm{S} 488 \mathrm{~W}=\mathrm{R} 247 \mathrm{~L}<\mathrm{S} 221 \mathrm{~L}<\mathrm{Q} 193 \mathrm{~A}<\mathrm{S} 452 \mathrm{Q}<$ $\mathrm{E} 237 \mathrm{D}<\mathrm{R} 247 \mathrm{~A}<\mathrm{P} 456 \mathrm{~A}=\mathrm{T} 453 \mathrm{~S}<\mathrm{S} 488 \mathrm{~A}=\mathrm{Q} 209 \mathrm{G})$.

Neol is primarily found in the Golgi, and a loss of its flippase activity should increase substrate lipids in the luminal leaflet of the Golgi and their ultimate exposure in the extracellular leaflet of the plasma membrane. Therefore, to test the influence of Neol mutations on substrate transport, we employed toxin sensitivity assays to measure the exposure of PS and PE on the plasma membrane extracellular surface (Fig. 5a). Papuamide A (Pap A) is a potent cytotoxic agent that binds specifically to PS, and duramycin binds specifically to PE. Both toxins produce pores in the membrane that kill sensitive cells on which their respective targets are exposed. WT yeast cells are relatively resistant to Pap $A$ and duramycin because most of their target lipids are restricted to the inner cytosolic leaflet of the plasma membrane. The neo1s strain expressing a WT copy of Neol was resistant to duramycin and papuamide relative to the neo1 1 any $1 \Delta$ strain, which exposed

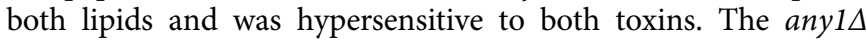
mutation suppresses neo1 $1 \Delta$ lethality but does not suppress the loss of PS and PE asymmetry caused by loss of Neol function ${ }^{44}$.
Relative to these two control strains, many of the Neol mutants displayed a significant loss of membrane asymmetry. We further explored the Neol mutants that had PE exposure and no apparent PS exposure by using higher concentrations of papuamide A to assess how well they maintained PS asymmetry (Fig. 5b, c). Neo1-S221L displayed a slight hypersensitivity to papuamide $\mathrm{A}$, but the rest of the mutants showed sensitivity indistinguishable from that of the WT.

The Neol mutants fell into three classes regarding substrate recognition (Fig. 5d). PS-specific mutants that expose PS but maintain near normal PE asymmetry (Q209G, S452Q, and T453S), PE-specific mutants that only expose PE (Q193A, S221L, R247A, and R247L), and mutants that expose both lipids. These substrate-specific, separation-of-function mutations strongly imply that the targeted residues are directly involved in substrate binding. For example, entry-site residues known to bind PS in ATP8A1-CDC50 correspond to the PSspecific Neo1 mutants. These results define residues crucial for $\mathrm{PE}$ recognition in Neol, and these residues are in the entry site (S221), transport path (Q193), and exit site (R247). We note 
a

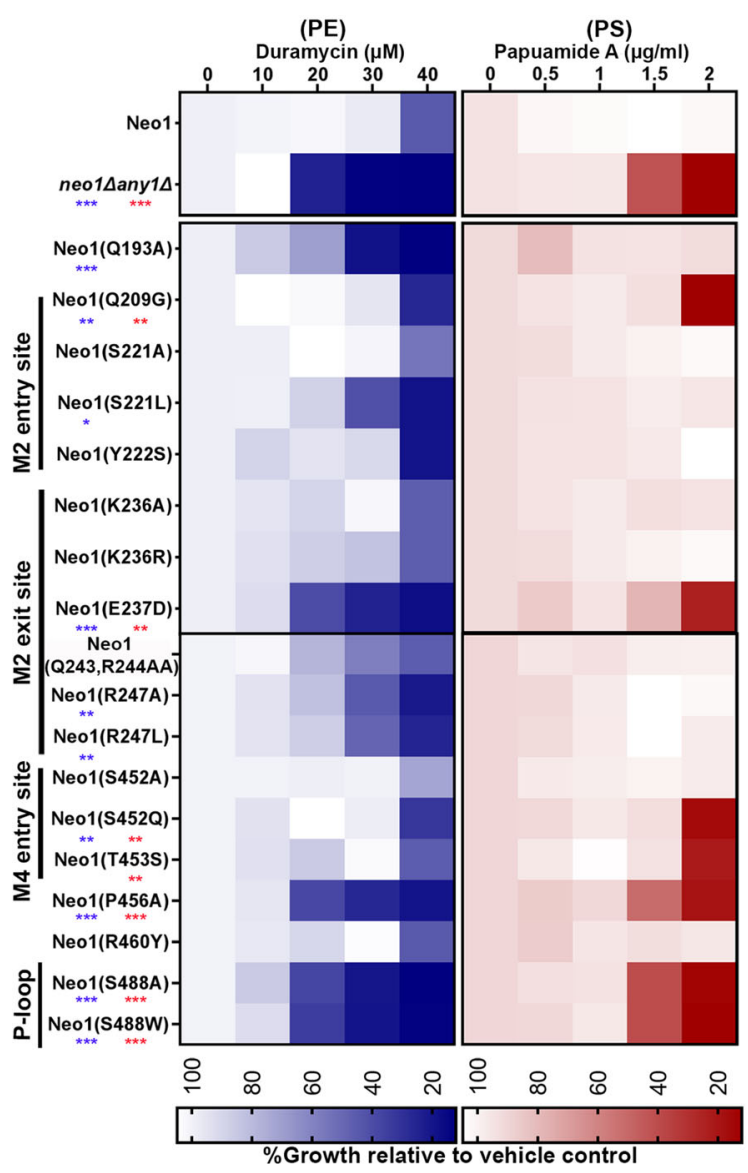

b
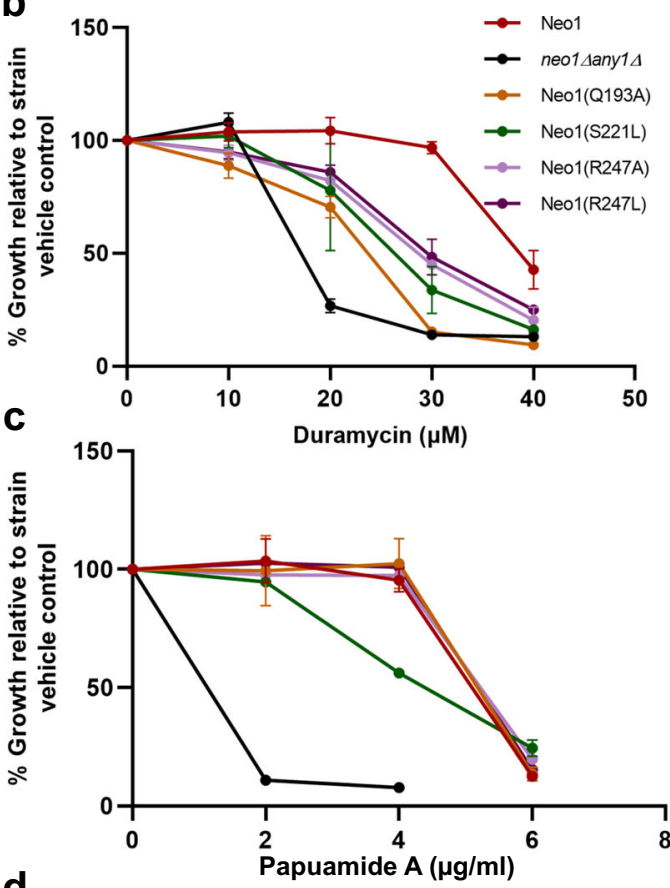

d

\begin{tabular}{|l|l|}
\hline PS Specific & Q209G, S452Q, T453S \\
\hline PE Specific & $\begin{array}{l}\text { Q193A, S221L, R247A, } \\
\text { R247L }\end{array}$ \\
\hline PS + PE & $\begin{array}{l}\text { E237D, S488A, S488W } \\
\text { P456A }\end{array}$ \\
\hline
\end{tabular}

Fig. 5 Neo1 substrate pathway mutations disrupt plasma membrane asymmetry. a Duramycin and papuamide A sensitivity of neo1 $\Delta$ strains expressing

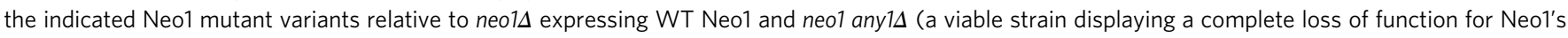
influence on membrane asymmetry). The blue heat map displays duramycin dose responses; growth inhibition at 20-30 $\mu \mathrm{M}$ indicates aberrant exposure of PE. The red heat map displays papuamide A dose responses; growth inhibition at $1.5-2 \mu \mathrm{g} / \mathrm{mL}$ indicates aberrant exposure of PS. The data represents growth relative to WT cells in absence of the drug. Two-way ANOVA was performed to test the variance and comparisons with WT Neo1 were made with Tukey's post hoc analysis $\left(n=3, \pm\right.$ standard deviation (SD)). ${ }^{\star} p<0.05,{ }^{\star \star} p<0.01,{ }^{\star \star \star} p<0.001$ (red for papuamide A and blue for duramycin). Neo1 (Y222S) is a gain-of-function mutation that weakly suppresses $d r s 2 \Delta^{57}$. b, c Neo1 mutants hypersensitive to only duramycin in (a) were tested at higher concentrations of papuamide A (up to $6 \mu \mathrm{g} / \mathrm{mL}$ ). All the values are an average of at least three biological replicates \pm standard deviation $(n=3, \pm S D$ ). d Table summarizing the effects of Neo1 mutations on membrane asymmetry.

that there was not a strong correlation between the effect of mutation on PE or PS asymmetry and growth (Figs. 4 and 5).

Mutations that decrease the transport of both PS and PE are not likely causing misfolding of Neol because these alleles fully support the growth of neo1 $\Delta$ cells (Fig. 4b). We tagged such Neo1 variants with GFP and examined their localization relative to mKate-Aur1, a Golgi marker. Mutations that significantly alter the structure of membrane proteins in the secretory pathway typically cause retention of the mutant protein in the endoplasmic reticulum $(\mathrm{ER})^{32,58}$. However, all of the Neol variants localized normally to the Golgi complex. In addition, we did not detect any significant difference in expression levels by immunoblotting for the GFP-tagged Neo1 variants (Supplementary Fig. 9). Thus, these mutations likely cause a loss of either substrate recognition or transport rather than a structural defect.

A comparison of the substrate-binding sites of lipid flippases. According to previous functional studies, each type of P4 ATPase may transport several different lipid substrates, but the preference is usually not equal ${ }^{35,51,52}$. We hypothesize that the substrate preference is likely determined by the chemical and electrophysiological properties of the substrate-binding sites. Having defined the substrate-binding sites in Neol via mutagenesis and functional assays, we compared the substrate entry sites of Neo1, Dnf1, Drs2, ATP8A1, and ATP11C (Fig. 6a), which are all surrounded by TMH1, TMH2, TMH4, and TMH6. We found that they all contained polar and non-charged residues such as Ser, Thr, Asn, and Gln. Structure-based sequence alignment showed that key residues at structurally equivalent positions were not well conserved in the primary sequence (Fig. 6b). The Neo1 functionally important dipeptide motif 209-QA-210 in TMH1 is analogous to GA in Dnf1 and Dnf2, QQ in Drs2 and ATP8A1, and the single residue $\mathrm{Q}$ in ATP11C. The Neo1 dipeptide motif 221-SY-222 in TMH2 is analogous to TT in Drs2 and ATP8A1, TS in ATP11C, LS in Dnf1, and FA in Dnf2. The Neo1 dipeptide motif 452-ST-453 becomes NN in ATP8A1, SN in Drs2, NF in ATP11C, and QS in both Dnf1 and Dnf2. Finally, the Neo1 residue Ala (A978) becomes an asparagine in all the other P4 ATPases compared here. The idea that these residues in the entry sites of the P4 ATPases play an important role in the lipid-transporting preference is underscored by previous 


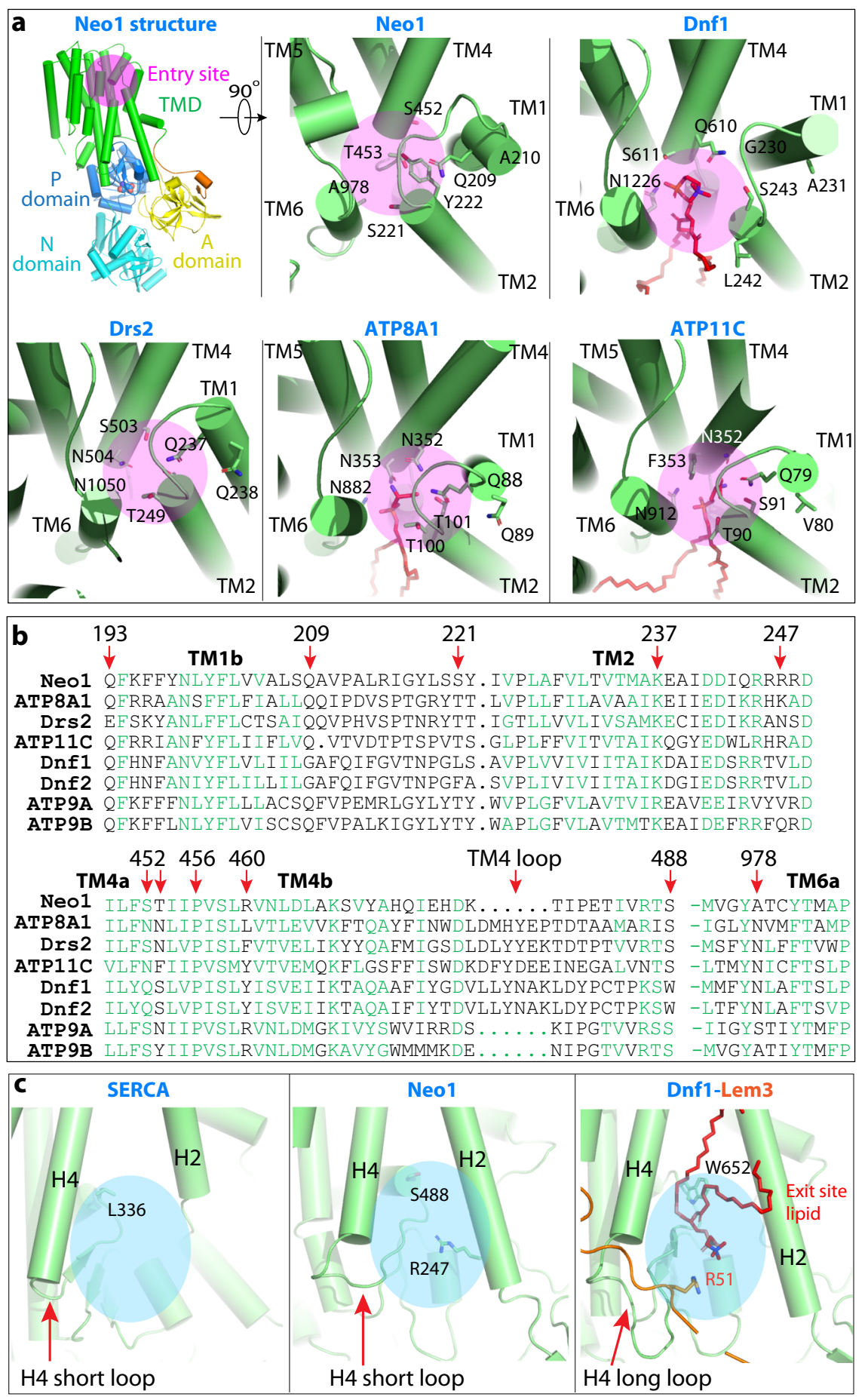

Fig. 6 Structural comparison of substrate entry and exit sites in Neo1 and other P4 ATPases. a Views of substrate entry sites in Neo1, Dnf1 (PDB ID 7KYC), Drs2 (PDB ID 6PSY), ATP8A1 (PDB ID 6K7M), and ATP11C (PDB ID 7BSV). The thermophilic Dnf1 (PDB ID 6LCP) is nearly identical to the S.c. Dnf1 and is not shown. Key residues and lipids (if present) are shown as sticks. The entry sites are highlighted by a magenta circle. $\mathbf{b}$ Structure-based sequence alignment of the S. cerevisiae Neo1, Dnf1, Dnf2, and Drs2, and the human ATP8A1, ATP11C, ATP9A, and ATP9B. Key residues involved in substrate lipid-binding are highlighted by red arrows. Conserved residues in well-aligned secondary structures are shown in green. The structure-based sequence alignment was performed online by ENDscript (https://espript.ibcp.fr/ESPript/ENDscript/). We modeled the ATP9A and ATP9B structures based on the Neo1 structure. c Comparison of substrate exit sites in SERCA (PDB ID 2AGV), Neo1, and Dnf1-Lem3 (PDB ID 7KYC). The blue circles mark the substrate exit sites.

mutational studies. In one study, mutations of the TMH1 key motif GA and the TMH4 motif QS of Dnf1/Dnf2 were shown to alter their activity for PC, PE, and GlcCer, and mutation of the TMH6 key reside N1226 of Dnf1 abolished the transport of all three substrates ${ }^{52}$. In another study, the TMH1 key motif QQ of Drs2 was shown to influence the specificity for PS transport ${ }^{49}$.

The substrate-binding exit site initially defined in Dnf1-Lem3 and Dnf2-Lem3 was unanticipated because it extends substantially out of the membrane plane into the cytosolic domains of 
these P4A ATPases. This exit site is structurally conserved in all P4 ATPase structures (Supplementary Fig. 6). It is formed from cytosolic extensions of TMH2 and TMH4 and a loop emanating from TMH4 that forms the membrane-proximal region of the $\mathrm{P}$ domain. We show in the current study that this cytosolically exposed lipid-binding site is functionally required for substrate transport in Neo1, a P4B ATPase. Comparison of the exit sites suggests a mechanism for how Neol functions without a $\beta$-subunit. The most membrane-distal residue forming the exit site in Dnf1-Lem3 is R51 in the cytosolic N-terminal tail of Lem3, analogous to R247 in Neo1 on the TMH2 side of the cleft (Fig. 6c). The $\beta$-subunit $\mathrm{N}$-terminal tail interacts with the loop from TMH4 that bends back towards the membrane to form part of the exit site (W652 in Dnf1), before starting the P-domain where the phosphorylated Asp resides. This TMH4 loop is much longer in the P4A ATPases than in the P4B ATPases and is nearly absent in P2 ATPases (e.g., SERCA) (Supplementary Fig. 10).

In summary, we have determined the structure of a singlesubunit lipid flippase and we suggest that P4B ATPases such as Neol arose first from cation transporters and gave rise to the P4A group with their new $\beta$-subunit requirement. The overall architecture and the substrate entry site of Neol are remarkably conserved in structure but, interestingly, not in amino acid sequence. Key differences between the P4A ATPases and P4B ATPases reflect regions important for $\beta$-subunit interaction, including the shorter extracellular loops in Neol, a shorter TMH4 cytosolic loop, and the ability to form the exit site in the absence of the $\beta$-subunit. Our extensive mutational studies identified key residues responsible for Neol's substrate preference. Structural comparison of Neo1 with other P4 ATPases revealed that the basic ATP-dependent lipid transport mechanism is highly conserved throughout evolution (Fig. 7). The capability and preference of the ATPases for transporting a multitude of lipid species are likely conferred by varying the amino acids of the key motifs in the otherwise structurally similar substrate entry and
(E1)

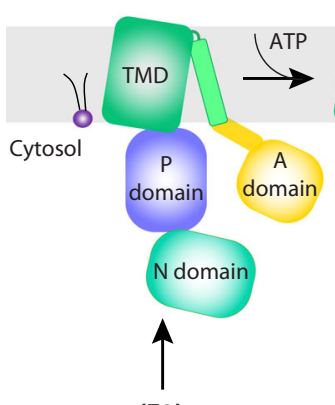

(E2)

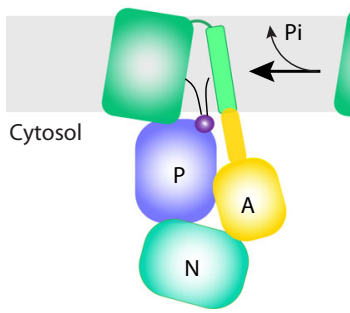

E1-ATP

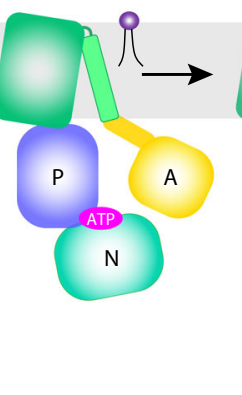

(E2P-Pi)

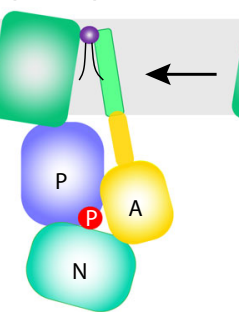

(E1-ADP)

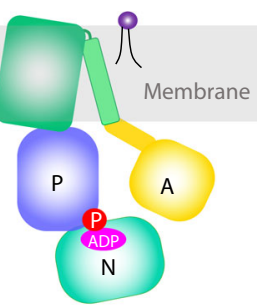

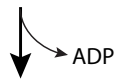

E2P

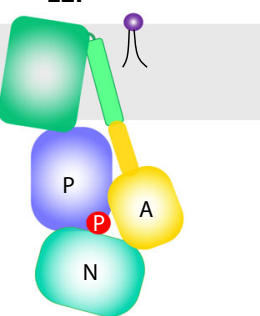

Fig. 7 A proposed lipid flipping mechanism by Neo1. The sketch highlights the six major states of the Post-Albers model, of which two are captured in this study. The four states not captured are labeled in paratheses. Despite being a single-subunit flippase, Neo1 follows the general mechanism proposed for the two-subunit P4 ATPases. The sketch also illustrates that the substrate lipid entry site dips into the transmembrane region and the exit site goes into the cytosolic region of the ATPase.

exit sites. Our work has advanced the mechanistic understanding of a large and often essential family of the P4 ATPases.

\section{Methods}

Expression and purification of Neo1. The NEO1 gene was cloned into pRS423 with a strong GAP promoter and an N-terminal triple FLAG tag. The construct was then transformed into $S$. cerevesiae. Yeast cells were grown in $2 \mathrm{~L} \mathrm{SD}-\mathrm{H}$ medium $(20 \mathrm{~g} / \mathrm{L}$ glucose, $6.7 \mathrm{~g} / \mathrm{L}$ yeast nitrogen base without amino acids, $0.04 \mathrm{~g} / \mathrm{L}$ adenine hemisulfate salt, and $1.92 \mathrm{~g} / \mathrm{L}$ yeast synthetic drop-out medium supplements without histidine) for about $20 \mathrm{~h}$, then transferred to $18 \mathrm{~L}$ of YPD medium for another $12 \mathrm{~h}$ before harvest. Cells were resuspended in lysis buffer (20 mM Tris$\mathrm{HCl}, \mathrm{pH} 7.4,0.2 \mathrm{M}$ sorbitol, $50 \mathrm{mM}$ potassium acetate, $2 \mathrm{mM}$ EDTA, and $1 \mathrm{mM}$ phenylmethylsulfonyl fluoride [PMSF]) and then lysed using a French press at 15,000 psi. The lysate was centrifuged at $10,000 \times g$ for $30 \mathrm{~min}$ at $4^{\circ} \mathrm{C}$. The supernatant was collected and centrifuged at $100,000 \times g$ for $60 \mathrm{~min}$ at $4{ }^{\circ} \mathrm{C}$. The membrane pellet was collected and then resuspended in buffer A containing $10 \%$ glycerol, $20 \mathrm{mM}$ Tris-HCl (pH 7.4), 1\% DDM, 0.1\% CHS, $0.5 \mathrm{M} \mathrm{NaCl}, 1 \mathrm{mM}$ $\mathrm{MgCl}_{2}, 1 \mathrm{mM} \mathrm{MnCl}, 1 \mathrm{mM}$ EDTA, and $1 \mathrm{mM}$ PMSF. After incubation for $30 \mathrm{~min}$ at $4{ }^{\circ} \mathrm{C}$, the mixture was centrifuged for $30 \mathrm{~min}$ at $120,000 \times g$ to remove the insoluble membrane. The supernatant was mixed with pre-equilibrated anti-FLAG

(M2) affinity gel (GenScript) at $4{ }^{\circ} \mathrm{C}$ overnight with shaking. The affinity gel was then collected and washed three times in buffer B (20 mM HEPES, pH 7.4, $150 \mathrm{mM}$ $\mathrm{NaCl}, 0.01 \% \mathrm{LMNG}, 0.001 \% \mathrm{CHS}$, and $1 \mathrm{mM} \mathrm{MgCl}_{2}$ ). The proteins were eluted with buffer B containing $0.15 \mathrm{mg} / \mathrm{mL} 3 \times$ FLAG peptide and were further purified in a Superose $610 / 300$ Increase gel filtration column in buffer C (20 mM HEPES, pH 7.4, $150 \mathrm{mM} \mathrm{NaCl}, 0.0025 \%$ LMNG, $0.00025 \% \mathrm{CHS}$, and $1 \mathrm{mM} \mathrm{MgCl}_{2}$ ). Finally, the purified proteins were assessed by sodium dodecyl sulfate-polyacrylamide gel electrophoresis gel and concentrated for cryo-EM analysis. Approximately, $20 \mu \mathrm{g}$ of $\mathrm{Neol}$ can be purified from $18 \mathrm{~L}$ of cells.

ATP hydrolysis assay. The ATPase activity assays were performed using BIOMOL Green Reagent (Enzo Life Sciences, Inc.) to measure released inorganic phosphate. The lipids PC, SM, PE, lyso-PS, and PS were solubilized with $20 \mathrm{mM}$ sodium cholate in $20 \mathrm{mM}$ HEPES, $\mathrm{pH} 7.4,150 \mathrm{mM} \mathrm{NaCl}$. Each reaction contained $0.025 \mathrm{mg} / \mathrm{mL}$ protein, $0.003 \%$ LMNG, 0.0003\% CHS, $20 \mathrm{mM}$ HEPES, pH 7.4, $150 \mathrm{mM} \mathrm{NaCl}, 10 \mathrm{mM} \mathrm{MgCl}_{2}$, and $0.25 \mathrm{mM}$ ATP. Reactions were carried out at $37^{\circ} \mathrm{C}$ for $15 \mathrm{~min}$ and then terminated immediately by addition of the reagent. After incubation of the mixture for $20 \mathrm{~min}$ at room temperature, the absorbance at $620 \mathrm{~nm}$ was measured using a microplate reader (SpectraMax M2e). The phosphate concentration was determined by calibration with the phosphate standard (BML-KI102).

Cryo-electron microscopy. To capture different states, the purified Neol was mixed with various solutions for $1 \mathrm{~h}$ on ice: E1-ATP, $5 \mathrm{mM} \mathrm{MgCl}_{2}$, and $2 \mathrm{mM}$ AMPPCP; E2P, $5 \mathrm{mM} \mathrm{MgCl}_{2}, 10 \mathrm{mM} \mathrm{NaF}$, and $2 \mathrm{mM} \mathrm{BeSO}_{4}$. After incubation, $2.5-\mu \mathrm{L}$ aliquots of purified Neol at a concentration of about $1 \mathrm{mg} / \mathrm{mL}$ were placed on glow-discharged holey carbon grids (Quantifoil Au R2/2, 300 mesh) and were flash-frozen in liquid ethane using an FEI Vitrobot Mark IV. Cryo-EM data were collected automatically with SerialEM in a $300-\mathrm{kV}$ FEI Titan Krios electron microscope with defocus values from -1.0 to $-2.0 \mu \mathrm{m}$. The microscope was operated with a K3 direct detector at a nominal magnification of 130,000 $\times$ and a pixel size of $0.413 \AA$ per pixel. The dose rate was 8 electrons per $\AA 2$ per s, and the total exposure time was $8 \mathrm{~s}$.

Cryo-EM image processing. Program MotionCorr $2.0^{59}$ was used for motion correction, and CTFFIND 4.1 was used for calculating contrast transfer function parameters ${ }^{60}$. All the remaining steps were performed using RELION-3 ${ }^{61}$. The resolution of the map was estimated by the gold-standard Fourier shell correlation at a correlation cutoff value of 0.143 .

For the Neo1 structure in the E2P state, we collected 4354 raw movie micrographs. A total of 2,315,630 particles were picked automatically. After 2D classification, a total of 2,068,707 particles were selected and used for 3D classification. Based on the quality of the four 3D classes, 1,279,510 particles were retained for further $3 \mathrm{D}$ reconstruction, refinement, and postprocessing, resulting in a 3.25- $\AA$ average resolution $3 \mathrm{D}$ map.

For the E1-ATP state, we collected 1175 raw movie micrographs. A total of 688,445 particles were picked automatically. After 2D classification, a total of 668,445 particles were selected and used for $3 \mathrm{D}$ classification. Based on the quality of the four 3D classes, 264,891 particles were selected for further 3D reconstruction, refinement, and postprocessing, resulting in the 5.64- $\AA$ average resolution $3 \mathrm{D}$ map.

For the E2P-transition state, we collected 5119 raw movie micrographs. A total of 3,037,926 particles were picked automatically. After 2D classification, a total of $2,661,522$ particles were selected and used for 3D classification. Based on the quality of the four $3 \mathrm{D}$ classes, 1,673,321 particles were selected for further $3 \mathrm{D}$ reconstruction, refinement, and postprocessing, resulting in the 3.08 - $\AA$ average resolution $3 \mathrm{D}$ map. 
Structural modeling, refinement, and validation. We first built the model of Neol in the E2P state at 3.2- $\AA$ resolution. We generated the initial model based on the structure of Dnf1 (PDB ID 7KYC) by SWISSMODEL (https:// swissmodel.expasy.org), and then manually corrected it in COOT and Chimera ${ }^{62,63}$. The complete Neol model was refined by real-space refinement in the PHENIX program and subsequently adjusted manually in COOT. Using the model of Neol in E2P as a reference, the model of Neol in the E2P-transition state at 3.1- $\AA$ resolution was built and refined using COOT, Chimera, and PHENIX. For the Neo1 in E1-ATP state at the 5.6- $\AA$ resolution, the model of Neo1 in E2P was split into TMD, A, N, and P domains, which were then fitted and refined into the map as the rigid body using COOT, Chimera, and PHENIX. Finally, all models were validated using MolProbity in PHENIX ${ }^{64,65}$. Structural figures were prepared in Chimera and PyMOL (https://pymol.org/2/).

Yeast strains and plasmid construction. The primers used in this study are listed in Supplementary Table 2. The strains and plasmids used in the study are listed in Supplementary Table 3 . All yeast culture reagents were purchased from SigmaAldrich and BD Scientific, and strains were grown in YPD or minimal selective media. Yeast transformation was performed using the standard LiAC-PEG method $^{66}$. For plasmid shuffling assays, 10 -fold serial dilutions starting from a cell suspension with a cell density of $\mathrm{OD}_{600}=1$ were spotted on synthetic defined media (SD) and SD-5-FOA and incubated at $30^{\circ} \mathrm{C}$ or temperatures indicated for at least $2 \mathrm{~d}$ before imaging. 5-FOA is converted to a toxic metabolite by the Ura3 protein and therefore cells retaining the URA3-marked plasmid cannot grow on 5 -FOA medium ${ }^{67,68}$. All images of yeast colonies are representative of three biological replicates (three independently isolated strains of the same genotype). DNA constructs and mutations were created by Gibson assembly and quick-change mutagenesis according to the manufacture instructions (using PfuTurbo; Agilent).

Toxin sensitivity assays. Papuamide A was a kind gift from Raymond Andersen from the University of British Columbia; duramycin was purchased from Sigma Aldrich. For toxin sensitivity assays, mid-log cells were diluted to $0.1 \mathrm{OD}_{600}$ in fresh YPD medium and $100 \mu \mathrm{L}$ of cells were distributed to each well of 96 wellplate with or without the toxin in $100 \mu \mathrm{L}$ of YPD. Toxin dilutions were calculated based on final concentrations in a total well volume of $200 \mu \mathrm{L}$. Plates were incubated at $30^{\circ} \mathrm{C}$ for $20 \mathrm{~h}$. Concentrations of the cells were measured in $\mathrm{OD}_{600} / \mathrm{mL}$ with a Multimode Plate Reader Synergy HT (Biotek). Growth relative to vehicle control (no drug) was used as $100 \%$ growth. All values are an average of at least three biological replicates \pm standard deviation.

Fluorescence microscopy. Strains expressing GFP-Neol and Aur1-mKate were grown to the mid-log phase in synthetic media. Cells were washed with fresh medium 3 times and resuspended in fresh SD medium. Cells were then mounted on glass slides and observed immediately at room temperature. Images were acquired using a DeltaVision Elite Imaging System equipped with a 100×,1.4 NA oil immersion objective lens followed by deconvolution using softWoRx software (GE Healthcare Cytiva, Pittsburgh, PA). Images were analyzed in FIJI (FIJI Is Just ImageJ) using the JaCOP (Just another Colocalization Plugin) add-on. Colocalization was quantified through the computation of the Manders' coefficient in $\mathrm{JaCOP}$ as the fraction of overlap between Neol and Aur1.

Reporting summary. Further information on research design is available in the Nature Research Reporting Summary linked to this article.

\section{Data availability}

The data that support this study are available from the corresponding authors upon reasonable request. The cryo-EM 3D maps and the corresponding atomic models of the Neol have been deposited at the EMDB database and the RCSB PDB with the respective accession codes of EMD-24413 and 7RD6 for E2P, EMD-24414 and 7RD7 for E2Ptransition, and EMD-24415 and 7RD8 for E1P-ATP. Source data are provided with this paper.

Received: 8 April 2021; Accepted: 28 September 2021; Published online: 13 October 2021

\section{References}

1. van Meer, G., Voelker, D. R. \& Feigenson, G. W. Membrane lipids: where they are and how they behave. Nat. Rev. Mol. Cell Biol. 9, 112-124 (2008).

2. Hankins, H. M., Baldridge, R. D., Xu, P. \& Graham, T. R. Role of flippases, scramblases and transfer proteins in phosphatidylserine subcellular distribution. Traffic 16, 35-47 (2015).

3. Doktorova, M., Symons, J. L. \& Levental, I. Structural and functional consequences of reversible lipid asymmetry in living membranes. Nat. Chem. Biol. 16, 1321-1330 (2020).
4. Emoto, K. et al. Redistribution of phosphatidylethanolamine at the cleavage furrow of dividing cells during cytokinesis. Proc. Natl Acad. Sci. USA 93, 12867-12872 (1996)

5. Emoto, K. \& Umeda, M. An essential role for a membrane lipid in cytokinesis Regulation of contractile ring disassembly by redistribution of phosphatidylethanolamine. J. Cell Biol. 149, 1215-1224 (2000).

6. Graham, T. R. Flippases and vesicle-mediated protein transport. Trends Cell Biol. 14, 670-677 (2004).

7. Xu, P., Baldridge, R. D., Chi, R. J., Burd, C. G. \& Graham, T. R Phosphatidylserine flipping enhances membrane curvature and negative charge required for vesicular transport. J. Cell Biol. 202, 875-886 (2013).

8. Montigny, C., Lyons, J., Champeil, P., Nissen, P. \& Lenoir, G. On the molecular mechanism of flippase- and scramblase-mediated phospholipid transport. Biochim. Biophys. Acta 1861, 767-783 (2016).

9. Yang, Y., Lee, M. \& Fairn, G. D. Phospholipid subcellular localization and dynamics. J. Biol. Chem. 293, 6230-6240 (2018).

10. Axelsen, K. B. \& Palmgren, M. G. Evolution of substrate specificities in the P-type ATPase superfamily. J. Mol. Evol. 46, 84-101 (1998).

11. Kuhlbrandt, W. Biology, structure and mechanism of P-type ATPases. Nat. Rev. Mol. Cell Biol. 5, 282-295 (2004).

12. Palmgren, M. G. \& Nissen, P. P-type ATPases. Annu. Rev. Biophys. 40, 243-266 (2011)

13. Palmgren, M. G. \& Axelsen, K. B. Evolution of P-type ATPases. Biochim. Biophys. Acta 1365, 37-45 (1998).

14. Bublitz, M., Morth, J. P. \& Nissen, P. P-type ATPases at a glance. J. Cell Sci. 124, 2515-2519 (2011).

15. Palmgren, M., Osterberg, J. T., Nintemann, S. J., Poulsen, L. R. \& LopezMarques, R. L. Evolution and a revised nomenclature of P4 ATPases, a eukaryotic family of lipid flippases. Biochim. Biophys. Acta Biomembr. 1861, 1135-1151 (2019).

16. Feng, Z. et al. CATP-8/P5A ATPase regulates ER processing of the DMA-1 receptor for dendritic branching. Cell Rep. 32, 108101 (2020).

17. McKenna, M. J. et al. The endoplasmic reticulum P5A-ATPase is a transmembrane helix dislocase. Science https://doi.org/10.1126/ science.abc5809 (2020).

18. Vrijsen, S. et al. ATP13A2-mediated endo-lysosomal polyamine export counters mitochondrial oxidative stress. Proc. Natl Acad. Sci. USA 117, 31198-31207 (2020).

19. van Veen, S. et al. ATP13A2 deficiency disrupts lysosomal polyamine export. Nature 578, 419-424 (2020).

20. Albers, R. W. Biochemical aspects of active transport. Annu. Rev. Biochem 36, 727-756 (1967)

21. Post, R. L., Hegyvary, C. \& Kume, S. Activation by adenosine triphosphate in the phosphorylation kinetics of sodium and potassium ion transport adenosine triphosphatase. J. Biol. Chem. 247, 6530-6540 (1972).

22. Toyoshima, C. \& Cornelius, F. New crystal structures of PII-type ATPases: excitement continues. Curr. Opin. Struct. Biol. 23, 507-514 (2013)

23. Dyla, M., Basse Hansen, S., Nissen, P. \& Kjaergaard, M. Structural dynamics of P-type ATPase ion pumps. Biochem. Soc. Trans. 47, 1247-1257 (2019).

24. Lopez-Marques, R. L., Gourdon, P., Gunther Pomorski, T. \& Palmgren, M The transport mechanism of P4 ATPase lipid flippases. Biochem. J. 477, 3769-3790 (2020)

25. Hiraizumi, M., Yamashita, K., Nishizawa, T. \& Nureki, O. Cryo-EM structures capture the transport cycle of the P4-ATPase flippase. Science 365, 1149-1155 (2019).

26. van der Mark, V. A., Elferink, R. P. \& Paulusma, C. C. P4 ATPases: flippases in health and disease. Int. J. Mol. Sci. 14, 7897-7922 (2013).

27. Andersen, J. P. et al. P4-ATPases as phospholipid flippases-structure, function, and enigmas. Front. Physiol. 7, 275 (2016)

28. Best, J. T., Xu, P. \& Graham, T. R. Phospholipid flippases in membrane remodeling and transport carrier biogenesis. Curr. Opin. Cell Biol. 59, 8-15 (2019).

29. Puts, C. F. et al. Mapping functional interactions in a heterodimeric phospholipid pump. J. Biol. Chem. 287, 30529-30540 (2012).

30. van der Velden, L. M. et al. Heteromeric interactions required for abundance and subcellular localization of human CDC50 proteins and class $1 \mathrm{P} 4$ ATPases. J. Biol. Chem. 285, 40088-40096 (2010).

31. Saito, K. et al. Cdc50p, a protein required for polarized growth, associates with the Drs2p P-type ATPase implicated in phospholipid translocation in Saccharomyces cerevisiae. Mol. Biol. Cell 15, 3418-3432 (2004).

32. Bai, L. et al. Transport mechanism of P4 ATPase phosphatidylcholine flippases. Elife https://doi.org/10.7554/eLife.62163 (2020)

33. Prezant, T. R., Chaltraw, W. E. Jr. \& Fischel-Ghodsian, N. Identification of an overexpressed yeast gene which prevents aminoglycoside toxicity. Microbiology 142, 3407-3414 (1996).

34. Wicky, S., Schwarz, H. \& Singer-Kruger, B. Molecular interactions of yeast Neolp, an essential member of the Drs2 family of aminophospholipid 
translocases, and its role in membrane trafficking within the endomembrane system. Mol. Cell Biol. 24, 7402-7418 (2004).

35. Wu, Y., Takar, M., Cuentas-Condori, A. A. \& Graham, T. R. Neol and phosphatidylethanolamine contribute to vacuole membrane fusion in Saccharomyces cerevisiae. Cell Logist. 6, e1228791 (2016).

36. Dalton, L. E., Bean, B. D. M., Davey, M. \& Conibear, E. Quantitative highcontent imaging identifies novel regulators of $\mathrm{Neol}$ trafficking at endosomes. Mol. Biol. Cell 28, 1539-1550 (2017).

37. Hua, Z. \& Graham, T. R. Requirement for neolp in retrograde transport from the Golgi complex to the endoplasmic reticulum. Mol. Biol. Cell 14, 4971-4983 (2003).

38. Takar, M., Wu, Y. \& Graham, T. R. The essential Neol protein from budding yeast plays a role in establishing aminophospholipid asymmetry of the plasma membrane. J. Biol. Chem. 291, 15727-15739 (2016).

39. Wehman, A. M., Poggioli, C., Schweinsberg, P., Grant, B. D. \& Nance, J. The P4-ATPase TAT-5 inhibits the budding of extracellular vesicles in C. elegans embryos. Curr. Biol. 21, 1951-1959 (2011).

40. Barbosa, S., Pratte, D., Schwarz, H., Pipkorn, R. \& Singer-Kruger, B. Oligomeric Doplp is part of the endosomal Neolp-Ysl2p-Arllp membrane remodeling complex. Traffic 11, 1092-1106 (2010).

41. Gillingham, A. K., Whyte, J. R., Panic, B. \& Munro, S. Mon2, a relative of large Arf exchange factors, recruits Dop1 to the Golgi apparatus. J. Biol. Chem. 281, 2273-2280 (2006).

42. McGough, I. J. et al. SNX3-retromer requires an evolutionary conserved MON2:DOPEY2:ATP9A complex to mediate Wntless sorting and Wnt secretion. Nat. Commun. 9, 3737 (2018).

43. van Leeuwen, J. et al. Exploring genetic suppression interactions on a global scale. Science https://doi.org/10.1126/science.aag0839 (2016).

44. Yamamoto, T., Fujimura-Kamada, K., Shioji, E., Suzuki, R. \& Tanaka, K. Cfslp, a novel membrane protein in the PQ-loop family, is involved in phospholipid flippase functions in yeast. G3 (Bethesda) 7, 179-192 (2017).

45. Bai, L. et al. Autoinhibition and activation mechanisms of the eukaryotic lipid flippase Drs2p-Cdc50p. Nat. Commun. 10, 4142 (2019).

46. Timcenko, M. et al. Structure and autoregulation of a P4-ATPase lipid flippase. Nature 571, 366-370 (2019)

47. He, Y., Xu, J., Wu, X. \& Li, L. Structures of a P4-ATPase lipid flippase in lipid bilayers. Protein Cell 11, 458-463 (2020).

48. Nakanishi, H. et al. Transport cycle of plasma membrane flippase ATP11C by Cryo-EM. Cell Rep. 32, 108208 (2020).

49. Baldridge, R. D. \& Graham, T. R. Two-gate mechanism for phospholipid selection and transport by type IV P-type ATPases. Proc. Natl Acad. Sci. USA 110, E358-E367 (2013).

50. Baldridge, R. D., Xu, P. \& Graham, T. R. Type IV P-type ATPases distinguish mono- versus diacyl phosphatidylserine using a cytofacial exit gate in the membrane domain. J. Biol. Chem. 288, 19516-19527 (2013).

51. Natarajan, P., Wang, J., Hua, Z. \& Graham, T. R. Drs2p-coupled aminophospholipid translocase activity in yeast Golgi membranes and relationship to in vivo function. Proc. Natl Acad. Sci. USA 101, 10614-10619 (2004).

52. Roland, B. P. et al. Yeast and human P4-ATPases transport glycosphingolipids using conserved structural motifs. J. Biol. Chem. 294, 1794-1806 (2019).

53. Jain, B. K., Roland, B. P. \& Graham, T. R. Exofacial membrane composition and lipid metabolism regulates plasma membrane P4-ATPase substrate specificity. J. Biol. Chem. 295, 17997-18009 (2020).

54. Baldridge, R. D. \& Graham, T. R. Identification of residues defining phospholipid flippase substrate specificity of type IV P-type ATPases. Proc. Natl Acad. Sci. USA 109, E290-E298 (2012).

55. Azouaoui, H. et al. High phosphatidylinositol 4-phosphate (PI4P)-dependent ATPase activity for the Drs2p-Cdc50p flippase after removal of its N- and C-terminal extensions. J. Biol. Chem. 292, 7954-7970 (2017).

56. Zhou, X., Sebastian, T. T. \& Graham, T. R. Auto-inhibition of Drs2p, a yeast phospholipid flippase, by its carboxyl-terminal tail. J. Biol. Chem. 288, 31807-31815 (2013).

57. Huang, Y., Takar, M., Best, J. T. \& Graham, T. R. Conserved mechanism of phospholipid substrate recognition by the P4-ATPase Neol from Saccharomyces cerevisiae. Biochim. Biophys. Acta Mol. Cell Biol. Lipids 1865, 158581 (2020).

58. Sun, Z. \& Brodsky, J. L. Protein quality control in the secretory pathway. J. Cell Biol. 218, 3171-3187 (2019)

59. Zheng, S. Q. et al. MotionCor2: anisotropic correction of beam-induced motion for improved cryo-electron microscopy. Nat. Methods 14, 331-332 (2017).

60. Mindell, J. A. \& Grigorieff, N. Accurate determination of local defocus and specimen tilt in electron microscopy. J. Struct. Biol. 142, 334-347 (2003).
61. Zivanov, J. et al. New tools for automated high-resolution cryo-EM structure determination in RELION-3. Elife https://doi.org/10.7554/eLife.42166 (2018).

62. Emsley, P., Lohkamp, B., Scott, W. G. \& Cowtan, K. Features and development of Coot. Acta Crystallogr. D. Biol. Crystallogr. 66, 486-501 (2010).

63. Pettersen, E. F. et al. UCSF Chimera-a visualization system for exploratory research and analysis. J. Comput. Chem. 25, 1605-1612 (2004).

64. Adams, P. D. et al. PHENIX: a comprehensive Python-based system for macromolecular structure solution. Acta Crystallogr. D Biol. Crystallogr 66, 213-221 (2010).

65. Chen, V. B. et al. MolProbity: all-atom structure validation for macromolecular crystallography. Acta Crystallogr. D Biol. Crystallogr 66, $12-21(2010)$

66. Gietz, R. D. \& Schiestl, R. H. Large-scale high-efficiency yeast transformation using the LiAc/SS carrier DNA/PEG method. Nat. Protoc. 2, 38-41 (2007).

67. Boeke, J. D., Trueheart, J., Natsoulis, G. \& Fink, G. R. 5-Fluoroorotic acid as a selective agent in yeast molecular. Genet. Methods Enzymol. 154, 164-175 (1987).

68. Takar, M., Huang, Y. \& Graham, T. R. The PQ-loop protein Anyl segregates Drs2 and Neo1 functions required for viability and plasma membrane phospholipid asymmetry. J. Lipid Res. 60, 1032-1042 (2019).

\section{Acknowledgements}

Cryo-EM images were collected in the David Van Andel Advanced Cryo-Electron Microscopy Suite at Van Andel Institute. We thank Gongpu Zhao and Xing Meng for facilitating data collection and David Nadziejka for the technical editing of this paper. We also thank Raymond Andersen from the University of British Columbia for the papuamide A. This work was supported by the U.S. National Institutes of Health (CA231466 to H.L. and GM107978 to T.R.G.) and the Van Andel Institute (to H.L.) Materials generated in this study are freely available by request to the corresponding authors.

\section{Author contributions}

L.B., B.K.J., T.R.G., and H.L. conceived and designed the experiments. L.B., B.K.J., Q.Y., and H.D.D. performed the experiments. MT generated strains and constructs. L.B., T.R.G., and H.L. analyzed the data. L.B., T.R.G., and H.L. wrote the paper with input from all authors.

\section{Competing interests}

The authors declare no competing interests.

\section{Additional information}

Supplementary information The online version contains supplementary material available at https://doi.org/10.1038/s41467-021-26273-0.

Correspondence and requests for materials should be addressed to Lin Bai, Todd $\mathrm{R}$. Graham or Huilin Li.

Peer review information Nature Communications thanks Yanyan Li and the other, anonymous, reviewer(s) for their contribution to the peer review of this work.

Reprints and permission information is available at http://www.nature.com/reprints

Publisher's note Springer Nature remains neutral with regard to jurisdictional claims in published maps and institutional affiliations.

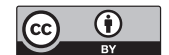

Open Access This article is licensed under a Creative Commons Attribution 4.0 International License, which permits use, sharing, adaptation, distribution and reproduction in any medium or format, as long as you give appropriate credit to the original author(s) and the source, provide a link to the Creative Commons license, and indicate if changes were made. The images or other third party material in this article are included in the article's Creative Commons license, unless indicated otherwise in a credit line to the material. If material is not included in the article's Creative Commons license and your intended use is not permitted by statutory regulation or exceeds the permitted use, you will need to obtain permission directly from the copyright holder. To view a copy of this license, visit http://creativecommons.org/ licenses/by/4.0/.

(C) The Author(s) 2021 